\title{
Evaluation of occult uterine leiomyosarcomas
}

\author{
Emrah Beyan ${ }^{1}$, Ahkam Göksel Kanmaz ${ }^{1}$, Abdurrahman Hamdi İnan², Volkan Karataşlı ${ }^{1}$, \\ Sadettin Oğuzhan Tutar ${ }^{3}$, Murat Alan ${ }^{1}$, Emrah Töz ${ }^{1}$, Muzaffer Sancı ${ }^{1}$ \\ ${ }^{1}$ Health Sciences University, Tepecik Education and Research Hospital, Department of Gynecology and Obstetrics, Izmir, Turkey \\ ${ }^{2}$ Ministry of Health Izmir Provincial Health Directorate Bornova Türkan Özilhan State Hospital, Izmir, Turkey \\ ${ }^{3}$ Ministry of Health Ardahan Provincial Health Directorate Ardahan State Hospital, Turkey
}

\begin{abstract}
Objectives: To determine the frequency of occult uterine leiomyosarcomas following hysterectomies and myomectomies performed for benign reasons at our clinic and to draw comparisons with similar studies in the literature.

Material and methods: All hysterectomies and myomectomies that have been performed for benign reasons at our clinic between 2010 and 2017 were retrospectively examined via the hospital's information system and the patients that were found to have leiomyosarcomas were analysed. The incidence of occult uterine leiomyosarcoma per 1000 surgeries at our clinic was calculated using the Wilson score interval.

Results: A total of 6,173 hysterectomies were performed, and occult uterine leiomyosarcoma was identified in 5 patients. The incidence of occult uterine leiomyosarcoma was calculated to be $0.08 \%$ ( $95 \% \mathrm{Cl} 0.03-0.018 \%$ ). Only 1 of the 771 patients who underwent myomectomy was identified with occult uterine leiomyosarcoma, making its incidence in myomectomy $0.12 \%$ (95\% Cl 0.02-0.073\%). When all the patients are considered, occult uterine leiomyosarcoma was identified in 6 of the 6,944 patients, and the general incidence of occult uterine leiomyosarcoma was calculated as $0.08 \%(95 \% \mathrm{Cl} 0.03-0.018 \%)$.

Conclusions: In our study, the incidence of occult uterine sarcoma following myomectomy and hysterectomy was found to be lower than that reported in the literature. The reason for this lower incidence includes not only genetic causes and racial differences but also preoperative imaging, endometrial and cervical sampling that is performed on every patient.
\end{abstract}

Key words: leiomyosarcoma; hysterectomy; myomectomy; myoma

Ginekologia Polska 2019; 90, 8: 433-437

\section{INTRODUCTION}

Hysterectomy is a commonly performed surgery in gynaecology, and it is reported that approximately 600,000 hysterectomies are performed annually in the South Korea, which is roughly similar to the number of such surgeries performed annually in the United States [1, 2]. A study conducted in Turkey in 2017 on secondary care hospitals affiliated with the Ministry of Health revealed that the total number of hysterectomies performed annually was more than 15,000 [3]. The large majority of hysterectomies are performed for benign reasons worldwide. According to a society-wide study carried out in the United States, the indications for hysterectomy include uterine fibroids in $51.4 \%$ of the cases, abnormal uterine bleeding in $41.7 \%$, endometriosis in $30 \%$ and uterine prolapse in $18.2 \%$ [4]. Another study in the United States determined that approximately 43,000 myomectomies are performed annually due to uterine fibroids, which is the most common reason for hysterectomy, whereas this figure is approximately 9,412 forTurkey [3,5].
Uterine sarcomas reportedly account for $1 \%$ of all gynaecological cancers, and 3-7\% of uterus-related cancers [6]. According to their histological classification, $40 \%$ of uterine sarcomas are carcinosarcomas, $40 \%$ are leiomyosarcomas, $10-15 \%$ are endometrial stromal sarcomas and 5-10\% are undifferentiated sarcomas [7]. Leiomyosarcomas, listed in this classification, show clinical findings similar to those of uterine fibroids, which leads to certain difficulties in the preoperative diagnosis and treatment of patients [7-9]. Although they are not specific, leiomyosarcomas are associated with several risk factors that are different from those associated with uterine fibroids, such as being from the black community, being diagnosed above the age of 60 years, use of tamoxifen or exposure to pelvic radiation, and retinoblastoma in childhood [10-13].

In recent years, there has been an increase in the number of hysterectomies and myomectomies performed worldwide through minimally invasive methods for indications of uterine fibroids, leading to a greater need for the morcella- 
tion of specimens. In this context, the U.S. Food and Drug Administration (FDA) 2014 has drawn attention to the possibility of occult gynaecological cancers, particularly uterine sarcomas that are associated with uterine fibroids [14]. Based on the possibility of uncontrolled spread within the abdomen in gynaecological cancers that could not be identified preoperatively, have been conducted following the aforementioned FDA report for determining the frequency of occult uterine sarcomas. A review of the literature shows that the frequency of occult uterine sarcomas tends to vary in the range of $0-0.89 \%[5,15]$.

\section{Objectives}

The present study aimed to determine the frequency of occult uterine leiomyosarcomas following hysterectomies and myomectomies performed for benign reasons at our clinic and to draw comparisons with similar studies in the literature.

\section{MATERIAL AND METHODS}

All hysterectomies and myomectomies that have been performed at Izmir University of Health Sciences Tepecik Training and Research Hospital between 2010 and 2017 were retrospectively examined via the hospital's information system. The preoperative diagnoses of all patients were examined. The study included patients who were operated for benign reasons, such as pelvic pain, leiomyoma, endometriosis, abnormal uterine bleeding and uterovaginal prolapse. For the past 10 years, our clinic has been collecting cervical smear samples for endometrial sampling purposes from all patients who have planned hysterectomy surgeries, with sample collection being performed at least 1 month before the date of surgery. Therefore, all patients included into the study had their preoperative assessment done according to these principles.

Patients with planned operations for diagnosed gynaecological or non-gynaecological cancers, patients with dysplasia or atypical endometrial hyperplasia, patients with prior surgeries performed for staging purposes, patients with suspicions of malignancy in their medical history and patients with hysterectomies performed for obstetric reasons were excluded from the study. The cases of uterine sarcomas that were initially not detected during preoperative examination but were subsequently identified as a result of postoperative histopathological examinations performed following hysterectomy and myomectomy surgeries were classified based on the guidelines of the World Health Organisation [16], and the patients that were found to have leiomyosarcomas were analysed.

The information of patients identified with occult uterine leiomyosarcomas was examined in detail through the hospital's information system and patient files. An analysis was performed on the patients' demographic characteristics, preoperative findings, primary surgical indication and type, weight of specimen, final pathology results, postoperative conditions and treatments and patient survival. The incidence of occult uterine leiomyosarcoma per 1000 surgeries at our clinic was calculated using the Wilson score interval.

Simultaneously, our results on occult uterine leiomyosarcoma were compared with those of suitable studies found by performing searches on PubMed using the MeSH terms 'Hysterectomy,',Myomectomy','Adenosarcoma,' 'Leiomyosarcoma' and 'Carcinosarcoma'.

\section{Statistical analysis}

The data were statistically analysed using SPSS 22.0 (SPSS Inc., Chicago, Illinois) software. The normality distribution of continuous variables was evaluated with a normality test that was suitable to the number of data collected. Mean \pm standard deviation or median (min. and max.) were used for descriptive continuous variables. The Mann-Whitney $U$ test was used for the comparison of variables lacking normal distribution, whereas the Student's t-test was used for the comparison of variables with normal distribution. Categorical variables were presented as number of cases and percentages. A value of $p<0.05$ was considered to be significant. EpiTools epidemiologic calculators (http://epitools.ausvet. com.au) were used for the Wilson score interval.

\section{RESULTS}

A total of 6,173 hysterectomies were performed at our clinic over 7 years, and occult uterine leiomyosarcoma was identified in 5 patients. The incidence of occult uterine leiomyosarcoma was calculated to be $0.08 \%(95 \% \mathrm{Cl}$ $0.03-0.018 \%$ ). Only 1 of the 771 patients who underwent myomectomy was identified with occult uterine leiomyosarcoma, making its incidence in myomectomy $0.12 \%$ (95\% Cl 0.02-0.073\%). When all the patients are considered, occult uterine leiomyosarcoma was identified in 6 of the 6,944 patients, and the general incidence of occult uterine leiomyosarcoma was calculated as $0.08 \%(95 \% \mathrm{Cl}$ $0.03-0.018 \%$ ), which is similar to its incidence in patients undergoing hysterectomy. Among the patients included in the study, 4,572 (65.8\%) underwent abdominal hysterectomy, $1,022(14.7 \%)$ underwent total laparoscopic hysterectomy, 579 (8.3\%) underwent vaginal hysterectomy and 771 (11.1\%) underwent myomectomy.

The most common indication of the patients who underwent abdominal and laparoscopic hysterectomy was symptomatic leiomyoma (39\%), whereas their second most common indication for hysterectomy was treatment-resistant menometrorrhagia (23\%). The most common indication of patients who underwent vaginal hysterectomy was pelvic organ prolapse (64\%). 
Among the patients included in our study, the primary surgical indication of those identified with occult uterine leiomyosarcoma was for symptomatic leiomyoma in all of them. Moreover, 2 patients also had an indication for treatment-resistant menometrorrhagia in addition to symptomatic leiomyoma.

The mean age of the patients included into the analysis was 48.63 years, whereas our subgroup analysis revealed that the patients who underwent abdominal hysterectomy, total laparoscopic hysterectomy, vaginal hysterectomy and myomectomy had a mean age of 48.91, 49.20, 61.20 and 37.28 years, respectively. The mean age of patients identified with occult uterine leiomyosarcoma was 46.5 years, which was lower than that of other patients. The patients' body mass indices varied between 22 and 28 . None of the patients identified with occult uterine leiomyosarcoma had any tamoxifen use prior to surgery. One patient used an oral contraceptive, whereas none of the other patients had any oral contraceptive use or received hormone replacement treatment. The uterus weight of the patients who underwent hysterectomy varied between 146 and $2500 \mathrm{~g}$. The patient whose uterus weighed $2500 \mathrm{~g}$ was found to be obese compared with the other patients, and it was understood that this patient did not attend her routine gynaecological examinations regularly.

The findings of the patients are summarised in Table 1. Six patients identified with occult uterine leiomyosarcoma had no pathological findings in their preoperative cervical smear and endometrial samples. Preoperative ultrasonography examination was performed on only 5 patients, whereas patient number 4 also had a magnetic resonance imaging performed in addition to ultrasonography, although their findings were not interpreted to be indicative of malignancy.

All of the patients were operated by gynaecology specialists under suitable conditions. Five patients had oophorectomies performed in addition to hysterectomy, and 1 patient only had a myomectomy surgery performed. Morcellation was not applied in any patient. In all patients, the operated piece was removed as a single whole piece, and we observed that, except for patient number 4, a frozen sample examination was not performed on the collected pieces, as there was no suspicion of malignancy. A frozen sample examination was performed on only 1 patient. The results of this examination were checked only according to the mitosis count, and the final pathological diagnosis of leiomyosarcoma was established in the final pathological examination.

Three patients identified with occult uterine leiomyosarcoma had stage 1c leiomyosarcoma, two had stage $1 \mathrm{~b}$ leiomyosarcoma and one had stage 2 leiomyosarcoma. Combined radiotherapy plus chemotherapy was used in the secondary treatment of 4 patients, whereas 1 patient received only radiotherapy and 1 received conservative treatment. None of the patients showed recurrence during their monitoring period, and all patients were still alive at the time this article was penned. The mean disease-free survival was determined as $60(40-86)$ months.

\section{DISCUSSION}

A total of 6,944 patients who underwent hysterectomy and myomectomy surgeries at our clinic for benign reasons within a seven-year period were included in the study. Occult uterine leiomyosarcoma was identified in 6 of the 6,944 patients, and the general incidence of occult uterine leiomyosarcoma was calculated as $0.08 \%(95 \% \mathrm{Cl}$ 0.03-0.018\%). Following the FDA's notable 2014 report, which presented a prevalence of $1 / 498(0.2 \%)$ for occult uterine leiomyosarcoma, there has been a visible increase in the literature in the frequency of studies conducted on this subject [17]. Many studies have reported the rates of occult uterine leiomyosarcoma at their own clinics, with incidences varying between $0 \%$ and $0.89 \%$. A summary of these studies is provided in Table 2.

In their 2008 study that focused solely on patients who underwent myomectomy, Sinha et al. [18] determined the incidence of occult uterine leiomyosarcoma to be $0.40 \%$, whereas Brohl et al. [19] identified this incidence to be $0.24 \%$. In our analysis, the rate of occult uterine leiomyosarcoma following myomectomy surgery was calculated

\begin{tabular}{|l|l|l|l|l|l|l|l|l|l|l|}
\multicolumn{9}{|l|}{ Table 1. Information of patients with occult uterine leiomyosarcoma } \\
\hline Patient & $\begin{array}{l}\text { Age } \\
\text { [years] }\end{array}$ & BMI & $\begin{array}{l}\text { Primary } \\
\text { diagnosis }\end{array}$ & Primary surgery & $\begin{array}{l}\text { Uterine } \\
\text { weight [g] }\end{array}$ & $\begin{array}{l}\text { Sarcoma } \\
\text { type }\end{array}$ & Stage & Treatment & Status & $\begin{array}{l}\text { Follow-up } \\
\text { [months] }\end{array}$ \\
\hline 1 & 52 & 28 & ULM & TAH & 241 & LMS & 1C & RT + CT & Live & 60 \\
\hline 2 & 47 & 29 & ULM + TRM & TAH & 146 & LMS & 1b & RT + CT & Live & 48 \\
\hline 3 & 54 & 31 & ULM & TAH & 610 & LMS & 2 & RT + CT & Live & 40 \\
\hline 4 & 47 & 38 & ULM & TAH & 2500 & LMS & 1C & RT & Live & 60 \\
\hline 5 & 47 & 27 & ULM + TRM & TAH & 469 & LMS & 1C & RT + CT & Live & 86 \\
\hline 6 & 32 & 22 & ULM & Myomectomy & 128 & LMS & 1b & None & Live & 72 \\
\hline
\end{tabular}

ULM - uterine leiomyoma; TRM — treatment-resistent menorrhagia; TAH — total abdominal hysterectomy; LMS — leiomyosarcoma; RT — radiotherapy; $\mathrm{CT}$ - chemotherapy 
Table 2. Comparison of occult uterine leiomyosarcoma studies

\begin{tabular}{|l|l|l|l|l|l|l|}
\hline Author & Year & $\begin{array}{l}\text { Centers that } \\
\text { include study }\end{array}$ & Operation type & Study duration & $\begin{array}{l}\text { Number of } \\
\text { patients }\end{array}$ & $\begin{array}{l}\text { Rate of Occult } \\
\text { LMS, } \\
\text { n (per 100) }\end{array}$ \\
\hline Parker et al. [26] & 1994 & Two & Hysterectomy, Myomectomy & $1988-1992$ & 1332 & $3(0.23)$ \\
\hline DiNapoli et al. [21] & 2018 & Single & Hysterectomy, Myomectomy & $2010-2014$ & 1959 & $4(0.20)$ \\
\hline Kundu et al. [20] & 2017 & Single & Hysterectomy, Myomectomy & $2004-2014$ & 2825 & $10(0.35)$ \\
\hline Seidman et al. [27] & 2012 & Single & Hysterectomy, Myomectomy & $2005-2010$ & 1091 & $2(0.18)$ \\
\hline Brohl et al. [19] & 2015 & Multiple & Myomectomy & $2005-2014$ & 2075 & $5(0.24)$ \\
\hline Sinha et al. [18] & 2008 & Single & Myomectomy & $1998-2005$ & 505 & $2(0.40)$ \\
\hline Multinu et al. [28] & 2019 & Multi & Hysterectomy & $1999-2013$ & 3759 & $4(0.11)$ \\
\hline Leung et al. [29] & 2008 & Single & Hysterectomy & $1996-2005$ & 1297 & $3(0.23)$ \\
\hline Ramm et al. [30] & 2012 & Multiple & Hysterectomy & $2004-2009$ & 708 & $1(0.14)$ \\
\hline Kho et al. [15] & 2016 & Single & Hysterectomy & $2000-2014$ & 10109 & $9(0.89)$ \\
\hline Yuk et al. [2] & 2016 & Multiple & Hysterectomy & $2010-2012$ & 12850 & $8(0.06)$ \\
\hline Theben et al. [31] & 2013 & Single & Hysterectomy & $2005-2010$ & 1584 & $2(0.13)$ \\
\hline Wright et al. [32] & 2014 & Multiple & Hysterectomy & $2006-2012$ & 36470 & none \\
\hline Wan et al. [33] & 2013 & Single & Hysterectomy & $2003-2011$ & 640 & $1(0.16)$ \\
\hline Current Study & & Single & Hysterectomy, Myomectomy & $2010-2017$ & 6944 & $6(0.08)$ \\
\hline LMS - & & & & & \\
\hline
\end{tabular}

LMS - leiomyosarcoma

to be $0.12 \%$. In addition to the specific geographical region where analyses are performed, racial and genetic differences are possibly the causes of these different results.

A review of the literature shows that while the incidence of occult uterine leiomyosarcoma in patients who undergo only hysterectomies can be $0.89 \%$ at the highest [15], the incidence of occult uterine leiomyosarcoma varies between $0.20 \%$ and $0.38 \%$ when all patients who underwent hysterectomy and myomectomy are included in the analysis $[20,21]$. In our study, the incidence of leiomyosarcoma was calculated to be $0.08 \%$, which is relatively lower than that reported in other studies. There are only a limited number of studies in Turkey regarding the incidence of occult leiomyosarcoma; however, a previous study in Turkey has reported an annual leiomyosarcoma incidence of $0.26 \%$ [22].

Currently, no tests that might be used to diagnose leiomyosarcoma preoperatively during the evaluation of patients for uterine leiomyoma are available. Unexplained pain and atypical vaginal bleeding in the presence of uterine leiomyoma along with a rapid increase in the leiomyoma's size can serve as warnings with regards to leiomyosarcoma $[7,23]$. As vaginal bleeding independently can be observed in submucosal myomas of any additional pathology and pain can also occur following a possible degeneration in the myomas, these two symptoms - vaginal bleeding and pain - have rather low value in preoperative differential diagnosis [24,25]. Parker et al have also reported that a rapid increase in the size of the uterus cannot be used in distinguishing leiomyosarcomas from leiomyomas [26].
As uterine leiomyosarcomas that have not been identified during preoperative differential diagnosis can possibly spread into the abdominal cavity, it is suggested that it is not suitable to perform power morcellation following minimally invasive procedures in the absence of adequate protective equipment for the intra-abdominal area $[18,20]$. The major limitation of this study was its retrospective nature. The fact that the hospital's information underwent a major overhaul in the 2000s and that we could not include patients admitted prior to this period considerably restricted our ability to increase our study sample size. Nevertheless, compared with the data obtained from single-centre studies performed in Turkey and worldwide, we believe that our study makes a marked contribution to the literature with its number of patients and the rates it determined. Simultaneously, we also believe that our study serves as an important source because it illustrates the situation in Turkey based on comparisons with other studies.

\section{CONCLUSIONS}

In our study, the incidence of occult uterine sarcoma following myomectomy and hysterectomy was found to be lower than that reported in the literature. We believe that the underlying reason for this lower incidence includes not only genetic causes and racial differences but also preoperative imaging and preoperative endometrial and cervical sampling that is performed on every patient. Although it might not seem cost-effective when viewed solely form a cost-effectiveness standpoint, we believe that detailed 
preoperative examination is useful given the possibility of medico-legal complications, additional treatment needs of patients and pain and anxiety the patients might experience. Conducting international multi-centre prospective studies on such patients will likely play a guiding role in determining the appropriate incidence of leiomyosarcoma and in identifying the proper measures against it.

\section{REFERENCES}

1. Tsui C, Klein R, Garabrant M. Minimally invasive surgery: national trends in adoption and future directions for hospital strategy. Surg Endosc. 2013; 27(7): 2253-2257, doi: 10.1007/s00464-013-2973-9, indexed in Pubmed: 23660720.

2. Yuk JS, Kim LY, Kim SH, et al. The Incidence of Unexpected Uterine Malignancy in Women Undergoing Hysterectomy for a Benign Condition: A National Population-Based Study. Ann Surg Oncol. 2016; 23(12):40294034, doi: 10.1245/s10434-016-5287-z, indexed in Pubmed: 27221362.

3. Alper M. Turkish Ministry of Health. Statistical report of State hospitals 2017. 2018. p. 78. https://dosyamerkez.saglik.gov. tr/Eklenti/21853,kamu-hastaneleri-istatistik-raporu--2017pdf.pdf?0.

4. Committee on Gynecologic Practice. Committee Opinion No 701: Choosing the Route of Hysterectomy for Benign Disease. Obstet Gynecol. 2017; 129(6): e155-e159, doi: 10.1097/AOG.0000000000002112, indexed in Pubmed: 28538495.

5. Desai VB, Wright JD, Schwartz PE, et al. Occult Gynecologic Cancer in Women Undergoing Hysterectomy or Myomectomy for Benign Indications. Obstet Gynecol. 2018; 131(4): 642-651, doi: 10.1097/AOG.0000000000002521, indexed in Pubmed: 29528920.

6. Major FJ, Blessing JA, Silverberg SG, et al. Prognostic factors in early-stage uterine sarcoma. A Gynecologic Oncology Group study. Cancer. 1993; 71(4 Suppl): 1702-1709, doi: 10.1002/cncr.2820710440, indexed in Pubmed: 8381710.

7. D'Angelo E, Prat J. Uterine sarcomas: a review. Gynecol Oncol. 2010; 116(1): 131-139, doi: 10.1016/j.ygyno.2009.09.023, indexed in Pubmed: 19853898.

8. Francis $M$, Dennis NL, Hirschowitz $L$, et al. Incidence and survival of gynecologic sarcomas in England. Int J Gynecol Cancer. 2015; 25(5): 850-857, doi: 10.1097/IGC.0000000000000443, indexed in Pubmed: 25853381.

9. AAGL Advancing Minimally Invasive Gynecology Worldwide. AAGL practice report: Morcellation during uterine tissue extraction. J Minim Invasive Gynecol. 2014; 21(4): 517-530, doi: 10.1016/j.jmig.2014.05.010, indexed in Pubmed: 24865630.

10. Lange S, Pluchino N, Fehlmann A, et al. Prevalence of undiagnosed uterine leiomyosarcoma in women undergoing hysterectomy or myomectomy for benign indications. Eur J Obstet Gynecol Reprod Biol. 2017; 216: 239-244, doi: 10.1016/j.ejogrb.2017.06.029, indexed in Pubmed: 28743407.

11. Fang $Z$, Matsumoto $S, A e ~ K$, et al. Postradiation soft tissue sarcoma: a multiinstitutional analysis of 14 cases in Japan. J Orthop Sci. 2004; 9(3): 242-246, doi: 10.1007/s00776-004-0768-5, indexed in Pubmed: 15168177.

12. Yildirim $Y$, Inal MM, Sanci M, et al. Development of uterine sarcoma after tamoxifen treatment for breast cancer: report of four cases. Int J Gynecol Cancer. 2005; 15(6): 1239-1242, doi: 10.1111/j.1525-1438.2005.00170.x, indexed in Pubmed: 16343223.

13. Toro JR, Nickerson ML, Wei MH, et al. Mutations in the fumarate hydratase gene cause hereditary leiomyomatosis and renal cell cancer in families in North America. Am J Hum Genet. 2003; 73(1): 95-106, doi: 10.1086/376435, indexed in Pubmed: 12772087.

14. Wood LN, Jamnagerwalla J, Markowitz MA, et al. Public Awareness of Uterine Power Morcellation Through US Food and Drug Administration Communications: Analysis of Google Trends Search Term Patterns. JMIR Public Health Surveill. 2018; 4(2): e47, doi: 10.2196/publichealth.9913, indexed in Pubmed: 29699965.

15. Hechanova ML, Lin K, Richardson DL, et al. Risk of Occult Uterine Sarcoma in Women Undergoing Hysterectomy for Benign Indications. Obstet
Gynecol. 2016; 127(3): 468-473, doi: 10.1097/AOG.0000000000001242, indexed in Pubmed: 26855091.

16. Kurman RJ, Carcangiu ML, Herrington CS YR. WHO Classification of Tumours of Female Reproductive Organs. In: 4th ed. Lyon: IARC; 2014. http://apps.who.int/bookorders/anglais/detart1.jsp?codlan=1\&codco $\mathrm{l}=70 \& \operatorname{cod} \mathrm{cch}=4006$

17. US Food and Drug Administration. Quantitative assessment of the prevalence of unsuspected uterine sarcoma in women undergoing treatment of uterine fibroids: summary and key findings. 2014. http://www.fda. gov/downloads/MedicalDevices/Safety/AlertsandNotices/UCM393589.pdf.

18. Sinha R, Hegde A, Mahajan C, et al. Laparoscopic myomectomy: do size, number, and location of the myomas form limiting factors for laparoscopic myomectomy? J Minim Invasive Gynecol. 2008; 15(3): 292-300, doi: 10.1016/j.jmig.2008.01.009, indexed in Pubmed: 18439500.

19. Brohl AS, Li Li, Andikyan V, et al. Age-stratified risk of unexpected uterine sarcoma following surgery for presumed benign leiomyoma. Oncologist. 2015; 20(4): 433-439, doi: 10.1634/theoncologist.2014-0361, indexed in Pubmed: 25765878.

20. Kundu S, Zachen M, Hertel H, et al. Sarcoma Risk in Uterine Surgery in a Tertiary University Hospital in Germany. Int J Gynecol Cancer. 2017; 27(5): 961-966, doi: 10.1097/IGC.0000000000000988, indexed in Pubmed: 28498262.

21. DiNapoli MN, Truong MD, Halfon JK, et al. Unsuspected Uterine Sarcoma in an Urban Hospital: Does Surgical Approach Matter? J Minim Invasive Gynecol. 2018; 25(3): 491-497, doi: 10.1016/j.jmig.2017.10.014, indexed in Pubmed: 29061369.

22. Gultekin M, Kucukyildiz I, Karaca MZ, et al. Trends of Gynecological Cancers in Turkey: Toward Europe or Asia? Int J Gynecol Cancer. 2017; 27(7): 1525-1533, doi: 10.1097/IGC.0000000000001026, indexed in Pubmed: 28708790.

23. Tropé CG, Abeler VM, Kristensen GB. Diagnosis and treatment of sarcoma of the uterus. A review. Acta Oncol. 2012; 51(6): 694-705, doi: 10.3109/0284186X.2012.689111, indexed in Pubmed: 22793037.

24. Han SC, Kim MD, Jung DC, et al. Degeneration of leiomyoma in patients referred for uterine fibroid embolization: incidence, imaging features and clinical characteristics. Yonsei Med J. 2013; 54(1): 215-219, doi: 10.3349/ymj.2013.54.1.215, indexed in Pubmed: 23225822.

25. Bharambe BM, Deshpande KA, Surase SG, et al. Malignant transformation of leiomyoma of uterus to leiomyosarcoma with metastasis to ovary. J Obstet Gynaecol India. 2014; 64(1): 68-69, doi: 10.1007/s13224-0120202-4, indexed in Pubmed: 24587612.

26. Parker WH, Fu YS, Berek JS. Uterine sarcoma in patients operated on for presumed leiomyoma and rapidly growing leiomyoma. Obstet Gynecol. 1994; 83(3): 414-418, indexed in Pubmed: 8127535.

27. Seidman MA, Oduyebo T, Muto MG, et al. Peritoneal dissemination complicating morcellation of uterine mesenchymal neoplasms. PLoS One. 2012; 7(11): e50058, doi: 10.1371/journal.pone.0050058, indexed in Pubmed: 23189178.

28. Multinu F, Casarin J, Tortorella L, et al. Incidence of sarcoma in patients undergoing hysterectomy for benign indications: a population-based study. Am J Obstet Gynecol. 2019; 220(2): 179.e1-179.e10, doi: 10.1016/j. ajog.2018.11.1086, indexed in Pubmed: 30447212.

29. Leung F, Terzibachian J-J, Aouar Z, et al. [Uterine sarcomas: clinical and histopathological aspects. Report on 15 cases]. Gynecol Obstet Fertil. 2008; 36(6): 628-635.

30. Ramm O, Gleason JL, Segal S, et al. Utility of preoperative endometrial assessment in asymptomatic women undergoing hysterectomy for pelvic floor dysfunction. Int Urogynecol J. 2012; 23(7): 913-917, doi: 10.1007/s00192-012-1694-2, indexed in Pubmed: 22398824.

31. Theben JU, Schellong ARM, Altgassen C, et al. Unexpected malignancies after laparoscopic-assisted supracervical hysterectomies (LASH): an analysis of 1,584 LASH cases. Arch Gynecol Obstet. 2013; 287(3): 455-462, doi: 10.1007/s00404-012-2559-0, indexed in Pubmed: 23053310.

32. Wright JD, Tergas Al, Burke WM, et al. Uterine pathology in women undergoing minimally invasive hysterectomy using morcellation. JAMA. 2014; 312(12): 1253-1255, doi: 10.1001/jama.2014.9005, indexed in Pubmed: 25051495.

33. Wan OYK, Cheung RYK, Chan SSC, et al. Risk of malignancy in women who underwent hysterectomy for uterine prolapse. Aust N Z J Obstet Gynaecol. 2013; 53(2): 190-196, doi: 10.1111/ajo.12033, indexed in Pubmed: 23316927. 